\title{
BMJ Open Prospective, observational, single-centre cohort study with an independent control group matched for age and sex aimed at investigating the significance of cholinergic activity in patients with schizophrenia: study protocol of the CLASH-study
}

\author{
Benedikt Schick (1D , ${ }^{1}$ Eberhard Barth, ${ }^{1}$ Benjamin Mayer, ${ }^{2}$ Claire-Louise Weber, ${ }^{3}$ \\ Theresa Hagemeyer, ${ }^{3}$ Carlos Schönfeldt-Lecuona ${ }^{3}$
}

To cite: Schick B, Barth E, Mayer B, et al. Prospective, observational, singlecentre cohort study with an independent control group matched for age and sex aimed at investigating the significance of cholinergic activity in patients with schizophrenia: study protocol of the CLASH-study. BMJ Open 2021;11:e050501. doi:10.1136/ bmjopen-2021-050501

- Prepublication history for this paper is available online. To view these files, please visit the journal online (http://dx.doi. org/10.1136/bmjopen-2021 050501).

Received 21 February 2021 Accepted 22 November 2021

D) Check for updates

(C) Author(s) (or their employer(s)) 2021. Re-use permitted under CC BY-NC. No commercial re-use. See rights and permissions. Published by BMJ.

For numbered affiliations see end of article.

\section{Correspondence to} Dr Benedikt Schick; benedikt-1.zujalovic@uni-ulm. de

\section{ABSTRACT}

Introduction Alterations in the cholinergic metabolism may cause various clinical symptoms of schizophrenia. In addition to the 'monoamine hypothesis,' neuroinflammation is also discussed as a cause of schizophrenia. To date, there has been no evidence of alterations in the central cholinergic transmitter balance in patients with schizophrenia under clinical conditions. By contrast, studies in critically ill patients have established the measurement of acetylcholinesterase activity as a suitable surrogate parameter of central cholinergic transmitter balance/possible pathophysiological changes. Butyrylcholinesterase activity has been established as a parameter indicating possible (neuro)inflammatory processes. Both parameters can now be measured using a point-ofcare approach. Therefore, the primary objective of this study is to investigate whether acetylcholinesterase and butyrylcholinesterase activity differs in patients with various forms of schizophrenia. Secondary objectives address the possible association between acetylcholinesterase and butyrylcholinesterase activity and (1) schizophrenic symptoms using the Positive and Negative Syndrome Scale, (2) the quantity of antipsychotics taken and (3) the duration of illness. Methods and analysis The study is designed as a prospective, observational cohort study with one independent control group. It is being carried out at the Department of Psychiatry and Psychotherapy III, UIm University Hospital, Germany. Patient enrolment started in October 2020, and the anticipated end of the study is in January 2022. The enrolment period was set from October 2020 to December 2021 (extension required due to SARS-CoV-2 pandemic). The sample size is calculated at 50 patients in each group. Esterase activity is measured on hospital admission (acute symptomatology) and after referral to a postacute ward over a period of three consecutive days. The matched control group will be created after reaching 50 patients with schizophrenia. This
Strengths and limitations of this study

- CLASH is the first study to investigate the clinical relevance of acetylcholinesterase and butyrylcholinesterase activity (BChE) measured using point-ofcare methods in patients with schizophrenia.

- The study design with a control group matched for age and sex as well as the planned subgroup analysis allow for a statistically correct analysis of the different aims of the study.

- The most significant limitation is the choice of $\mathrm{BChE}$ as a marker of neuroinflammation, since it is unclear whether BChE activity is specific enough to identify the neuroinflammatory component as a cause of schizophrenia.

will be followed by a comprehensive statistical analysis of the data set.

Ethics and dissemination The study was registered prospectively in the German Clinical Trials Register (DRKSID: DRKS00023143,URL: https://www.drks.de/drks_ web/navigate.do? navigationld=trial.HTML\&TRIAL ID $=$ DRKS00023143) after approval by the ethics committee of the University of UIm, Germany Trial Code No. 280/20.

Trial registration number DRKS00023143; Pre-results.

\section{INTRODUCTION}

Schizophrenia is a severe psychiatric illness with a lifetime prevalence of $1.0 \%{ }^{1}$ The suicide risk of patients with this condition is significantly higher than that of the general population. ${ }^{2}$ Among other things, this is attributable to the usually high intensity of patient suffering and delusions. In terms of clinical aspects, symptoms can be categorised as 'positive' and 'negative'. ${ }^{3}$ Positive 
symptoms include delusional paranoid experiences, hallucinations, distortions of self-experience, thought disorders and hyperkinetic-catatonic symptoms. Negative symptoms include anhedonia, asociality, avolition, apathy and a blunted affect, maybe even hypokinetic-catatonic symptoms. ${ }^{45}$ The International Statistical Classification of Diseases, 10th Revision and Related Health Problems (chapter V (F)) of the WHO differentiates various forms of schizophrenia in Section F2, similarly to the Diagnostic and Statistical Manual of Mental Disorders (DSM) used in English-speaking countries. Despite all of the diagnostic criteria specified, schizophrenia remains a diagnosis of exclusion. ${ }^{6}$

The elusive nature of the disease when it comes to diagnosis is also reflected in the various pathophysiological explanatory hypotheses for schizophrenia. As a currently accepted doctrine, the dopamine hypothesis holds that a disturbance in the central dopamine homeostasis leads to the typical hallucinatory symptoms of schizophrenia. ${ }^{7}$ While positive symptoms are attributed to hyperactive dopaminergic mesolimbic regions, negative symptoms might be associated with a deficiency in dopaminergic activity in the frontal mesocortical regions. This is also the rationale for pharmacological treatment with dopamine antagonists when treating hallucinations and delusions. ${ }^{8}$ However, the dopamine hypothesis is of limited validity, as it cannot adequately explain the clinical manifestations of cognitive impairment such as learning and memory deficits. $^{9}$

\section{Cholinergic system, acetylcholinesterase activity and schizophrenia}

In patients with schizophrenia, imaging and postmortem studies have postulated the theory that alterations in the cholinergic transmitter balance act as a partial aspect of pathophysiologic changes. ${ }^{10-13}$ In particular, reduced activity of the muscarinic M1-M4 receptors and changes at the nicotinic n-alpha-7-acetylcholine receptor were identified. ${ }^{14-16}$ Among other things, acetylcholine mediates perception, attention and cognitive performance via central muscarinergic receptors. By binding to nicotinic acetylcholine receptors located in the central nervous system (CNS), it also assists with the modulation of the neuronal excitation level and is thus involved in learning and memory competence. Alterations in in the central cholinergic transmitter balance are often reflected in symptoms such as learning, memory, and attention disorders, which can manifest clinically in the form of delirium and dementia syndrome, but also in patients with schizophrenia. It is currently not possible to assess cholinergic transmitter balance under clinical conditions because of the short half-life of its transmitter, acetylcholine. As a surrogate marker of the central cholinergic transmitter balance, erythroyctic acetylcholinesterase (AChE) activity offers an approximate reflection of the activity of AChE in the CSF (cerebrospinal fluid) and has become established as such, especially in the field of critical care medicine. ${ }^{17-19}$
The negative symptomatology can be explained by a disturbance in the central cholinergic transmitter homoeostasis. ${ }^{20}$ Case-control studies focusing on indirect parasympathomimetics such as donepezile, rivastigmine and arecoline, which can cross into the CNS, support this hypothesis. ${ }^{21-26}$ Studies in critically ill, delirious patients as well as patients with Alzheimer's disease, support the theory of central cholinergic transmitter deficiency based on comparable clinical symptoms such as learning, memory and attention deficits. ${ }^{17} 182728$ Since most schizophrenic spectrum disorders are usually chronic and sometimes lifelong, patients are at a high risk of suffering from iatrogenic central cholinergic dysfunction due to the long-term use of potentially anticholinergic drugs such as most antipsychotics, benzodiazepines and antidepressants. ${ }^{29}{ }^{30}$ Schizophrenic patients are often heavy smokers. ${ }^{31}{ }^{32}$ It is postulated that the heavy consumption of nicotine is a kind of self-therapy on the part of the patient aimed at compensating for a possible cholinergic deficit, be it medicinal or diseaserelated. ${ }^{33-36}$ The type and amount of daily nicotine consumption must, therefore, be considered in the context of measuring acetylcholinesterase and butyrylcholinesterase activity (BChE) in both patients and the control group, as falsely high measured values may occur. The study design does not include an analysis of genetic factors that may be associated with alterations in central cholinergic transmission in patients with schizophrenia. ${ }^{163738}$ However, genetic factors should be considered in the discussion of pathophysiological alterations in central cholinergic transmitter balance as a cause of cholinergic deficit in schizophrenics. To date, the evidence available regarding disturbances of the central cholinergic transmitter balance or cholinergic neurons in patients with schizophrenia is limited. A number of systematic reviews address the need for research in this area ${ }^{38-40}$ Therefore, unlike previous approaches, this study attempts to objectify a change in the central cholinergic metabolism on the basis of point-of-care measurements of erythrocytic AChE activity.

\section{Neuroinflammation and BChE}

The neuroinflammation theorem is another explanatory approach to the pathogenesis of schizophrenia spectrum disorders. ${ }^{41-46}$ It is common knowledge that central cholinergic metabolism interacts with the immune response via the cholinergic-anti-inflammatory reflex arc. ${ }^{47}$ In contrast to neuronally expressed AChE, BChE is predominantly synthesised by immunologically active glial cells in the CNS, which highlights its importance as an inflammatory marker. ${ }^{4-51}$ Various studies have shown dysregulation of proinflammatory and anti-inflammatory molecules in patients with schizophrenia. ${ }^{52-54}$ Neuroimaging studies show partial activation of microglia in these patients. BChE activity, as an essential parameter of this study, is produced predominantly by immunologically 
active glial cells in the CNS. Studies in critically ill patients have already established a decrease in BChE activity as both an inflammatory marker and an outcome parameter. ${ }^{495} 56$ Contrary to a study by Mabrouk we assume that a decrease rather than an increase in plasma BChE activity is observed in schizophrenic patients where (neuro)inflammation is considered as a possible cause. ${ }^{57}$ The reason for our assumption is that the BChE activity is shown to correlate negatively with the inflammatory level. ${ }^{49-60}$ Furthermore, in our study, a 3-day measurement sequence is performed that takes into account the inter- and intraindividual variability of both BChE and AChE activity. Considering the interaction of cholinergic metabolism with the immune system, referred to as the cholinergic anti-inflammatory reflex arc, it is obvious why the joint measurement of both AChE and BChE activity may be of interest in patients with schizophrenia. Based on the research of Tracey, the following mechanistic consideration describes the cholinergic-anti-inflammatory reflex: Proinflammatory molecules lead to activation of the vagal afferents. As a result, increased acetylcholine is released via vagal afferents. ACh binds to the alpha-7n-acetylcholine receptor expressed on immunologically active cells. The activated immune cells release more antiinflammatory molecules and thereby control inflammation. ${ }^{61}$ Various studies have shown that disruption of this reflex arc results in excessive inflammation. By contrast, a significant anti-inflammatory response could be achieved via electrical or pharmacological stimulation of the vagus nerve. $^{62} 63$

Taking into consideration the aforementioned theorems, the aim of this study is to evaluate whether AChE and BChE activity are altered in patients with schizophrenia compared with a healthy control group and whether, as a secondary endpoint, changes in AChE and/ or BChE activity correlate with different clinical manifestations of schizophrenia as a result of subgrouping within the schizophrenic patient collective.

The ability to measure both AChE and BChE activity using point-of-care diagnostic methods offers the option to establish a systematic survey of patients with schizophreniform disorders in the clinical setting.

\section{METHODS/DESIGN}

The CLASH study is designed as a prospective, observational, single-centre cohort study with one independent control group, matched by age and sex. Patient enrolment started in October 2020, and the anticipated end of the study is in January 2022. The enrolment period was set from October 2020 to December 2021. At the time of data collection, all patients were receiving inpatient or outpatient treatment at the Department of Psychiatry and Psychotherapy III, Ulm University Hospital, Germany.

\section{Study population}

All patients of the Department of Psychiatry and Psychotherapy III at the University Hospital Ulm, Germany, whose disorder is classified as schizophrenia spectrum on hospital admission according to the current version of DSM-5 are potentially eligible for inclusion. In the course of data evaluation, a distinction is then made between whether the diagnosis is an initial one or whether the patient already suffers from a schizophreniform disorder. The control group is recruited from healthy volunteers who are matched for sex and age. The following inclusion and exclusion criteria apply to patients with schizophrenia.

\section{Inclusion criteria}

- Age $\geq 18$ years.

- Schizophrenia classified by DSM (pre-existing or first episode).

- Verbal and written patient consent before the start of measurements.

\section{Exclusion criteria}

- Age $<18$ years.

- Cognitive impairment that makes study participation impossible.

- Severe visual and hearing impairments.

- Patients who are unable to give informed consent.

- Insufficient German language skills, making communication impossible.

Inclusion and exclusion criteria for the matched group of healthy subjects are defined as follows:

- Age $\geq 18$ years.

- Anamnestic exclusion of a previous psychiatric disorder.

- Verbal and written volunteer informed consent before the start of measurements.

The exclusion criteria are the same as those for patients with schizophrenia.

The following patient-related data were collected during the hospital treatment:

- Age at enrolment.

- Sex.

- Length of stay.

- Continuous and on-demand medication, number of potential anticholinergic medication according to Anticholinergic Cognitive Burden (ACB) scale.

- Nicotine consumption (pack-years) and current number of cigarettes smoked per day actually/transdermal nicotine application in $\mathrm{mg}$.

- Time of first appearance of the disease.

- Results of psychological test examinations PANNS, Mini-Mental State Examination (MMSE), SelfEvaluation of Negative Symptoms (SNS).

- List of predictors of schizophrenia.

\section{Measurement of AChE and BChE activity}

Erythrocytic AChE and plasma BChE activity is measured using point-of-care diagnostics (LISA-ChE, 
Köhler Chemie-CE certified). This is a colorimetric procedure based on the Ellman method modified by Worek et al. Measurement of erythrocytic AChE activity is performed by hydrolysis of the thioester acetylthiocholine. The thioalcohol formed is reacted with Ellmans reagent (DTNB 5,5'-dithiobis-2-nitrobenzoic acid). The 3-carboxy-4-nitrobenzenethiolate anion (TNB) anion obtained turns yellow in a neutral or basic solution and can be determined photometrically at $470 \mathrm{~nm}$ by absorbance change. The result is expressed in U/ gHb. Similarly to the measurement described above, s-butyrylthiocholine iodide is used as substrate for the measurement of BChE activity. The result is expressed in U/1. For a more detailed description of the chemical background, we refer the reader to the work of Worek et al. ${ }^{64}$ The measurement itself is carried out by making a minimal stab incision on a fingertip with a lancet once a day, similar to the measurement of blood

Table 1 Overview of the study procedure

\section{Selection of eligible patients}

Reason for admission:

- Exacerbation of the disease

Symptoms:

- Severe disorganisation in thinking and acting

External or internal hazard
Reason for admission:

- Exacerbation of the disease

Symptoms:

No severe disorganisation in thinking and acting

- No external or internal hazard
Reason for admission:

- Chronic schizophrenia and poor functional level

Symptoms:

- Inability to maintain existing levels of functioning in the activities of daily life

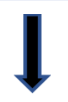

Admission ward:
- Acute closed ward

Admission ward:

Admission ward:

- Open ward

- Outpatient clinic

\section{Time of measurement of $\mathrm{AChE}$ and BChE activity}

First measurement:

- EDTA blood (1x) as part of the admission laboratory tests

- Additional tube but no additional venous puncture
First measurement:

- EDTA blood $(1 \times)$ as part of the admission laboratory tests

- Additional tube but no additional venous puncture

\section{First measurement:}

- EDTA blood (1x) as part of the admission laboratory tests

- Additional tube but no additional venous puncture

Patient education:

- Takes place before the subsequent measurements.

- If the patient is capable of education or has a legal guardian, oral consent as well as the patient's ability to cooperate is always a prerequisite.

- If participation in the study is declined, no further data will be collected.

The measured values from the admission laboratory tests are discarded.
Subsequent measurements:
Subsequent measurements:
Day 1 to day 3:
Day 1 to day 3:
Subsequent measurements:
- If consent is obtained, measurement taken on the acute care ward; otherwise after transfer
- Open ward or outpatient clinic
Day 1 to day 3 :
- Outpatient clinic

Collection of patient-related data (age, sex, height, weight, BMI, nicotine consumption, school-leaving qualification etc):

- Takes place after completion of the AChE and BChE measurements.

- Written documentation in the sample sheets

- Creation of a database on a computer belonging to the university network (no patient-specific data on our personal laptops)

Collection of disease specific data:

- Takes place in consultation with the patient on any day of the 3-day measurement sequence.

- Checklist of predictors of schizophrenia

- Positive and Negative Syndrome Scale

- Mini-Mental Status Test

- Self-reported negative syndromes

Further data to be collected:

- Routine laboratory parameters

- No and type of drugs prescribed before admission to the hospital (long-term medication)

- No and type of anticholinergic drugs

The red color indicates patients with further treatment in the acute closed war.The blue color indicates patients with further treatment in an outpatient clinic.

AChE, acetylcholinesterase activity; BChE, butyrylcholinesterase activity; BMI, body mass index. 
Table 2 Sample size calculation

\section{Two-group t-test of equal means}

\begin{tabular}{lllll}
\hline & \multicolumn{2}{l}{ AChE activity } & \multicolumn{2}{l}{ BChE activity } \\
\hline $\begin{array}{l}\text { Test significance } \\
\text { level } \alpha\end{array}$ & 0.050 & 0.050 & 0.050 & 0.050 \\
\hline Two sided & 2 & 2 & 2 & 2 \\
\hline Group 1 mean, $\mu_{1}$ & 34.30 & 34.30 & 2695.60 & 2596.60 \\
\hline Group 2 mean, $\mu_{2}$ & 32.60 & 30.90 & 2560.80 & 2426.00 \\
$\begin{array}{l}\text { Difference in means, } \\
\mu_{1}-\mu_{2}\end{array}$ & 1.70 & 3.40 & 134.80 & 269.60 \\
Common SD, $\sigma$ & 2.50 & 2.50 & 150.0 & 150.0 \\
$\begin{array}{l}\text { Effect size, } \delta=\left(\mu_{1}-\right. \\
\left.\mu_{2}\right) / \sigma\end{array}$ & 0.680 & 1.360 & 0.899 & 1.797 \\
Power (\%) & 80 & 80 & 80 & 80 \\
n per group & 35 & 10 & 21 & 6 \\
\hline
\end{tabular}

AChE, acetylcholinesterase activity; BChE, butyrylcholinesterase activity.

glucose. Every $2 \times 10 \mu \mathrm{L}$ of the blood drops collected are transferred to a heparin coated glass capillary. The blood is then transferred into reagent containers as directed in the manufacturer's instructions. A result is available after 5 min.

\section{Objectives of the study}

The primary objective of the study is to investigate whether:

1. AChE activity differs in patients with various forms of schizophrenia compared with a healthy population matched for age and sex.

2. BChE activity differs in patients with various forms of schizophrenia compared with a healthy population matched for age and sex.

The following questions have been defined as secondary objectives:

1. Is there a statistically significant correlation between AChE and/or BChE activity and the number of points achieved in the Positive and Negative Syndrome Scale (PANSS)?

2. Is there a correlation between the course of AChE, BChE activity over time and the quantity of antipsychotics (total dose, dose per 24 hours)?

3. Does the length of illness (related to the initial diagnosis of schizophrenia) correlate with the intraindividual and interindividual course of AChE, BChE activity?

\section{Patient recruitment and study procedures}

Table 1 provides an overview of the study procedure. After screening for inclusion and exclusion criteria, the reason for admission is documented for all potentially eligible patients, with a focus on disease length and symptoms. This is intended for later retrospective subgroup analysis. Based on the admission laboratory tests conducted, baseline measurement of AChE and BChE activity is performed from EDTA blood. All further measurements of esterase activities are taken from capillary blood. If patients remain in the closed acute ward, the 3-day measurement cycle (1 measurement each of $\mathrm{AChE}$ and BChE activity daily) starts in this ward. If it is expected that patients can be transferred to an open ward or to outpatient hospital care within 3-5 days after admission, the 3-day measurement cycle begins on these wards. The latency between the baseline measurement and the 3-day measurement cycle is intended in particular to record the effects of therapy initiation or drug changes on the cholinergic transmitter balance. All drugs administered are examined for their potential anticholinergic effect according to the classification on the ACB Scale. The intraindividual and interindividual course can be depicted most accurately over several days.

The clinical rating scales are collected as follows: The MMSE score is calculated on the first day of the 3-day measurement series. On the second day of the 3-day measurement series, possible predictors such as positive family history, previous brain disorders, maternal drug or alcohol use during pregnancy, psychosocial stress factors, expressed emotions and substance abuse as mentioned in the S3 Guideline on Schizophrenia published by the German Society for Psychiatry and Psychotherapy, Psychosomatics, among others, are recorded. ${ }^{65}$ The SNS is subsequently provided to the patient, who is asked to complete the form and return on the next day of measurement.

\section{Statistical analysis}

Data are collected in Microsoft Excel 2019 (Microsoft, Redmond, Washington, USA) and statistically analysed using Sigma Plot V.14 for Windows (Systat Software, Erkrath, Germany) and SAS V.9.4 (SAS Institute). Quantitative data were expressed as medians with interquartile ranges and were compared for nonparametric distributions using the Wilcoxon matched pairs test. For the primary endpoint, we assume that a change in AChE, BChE activity is detectable between patients with schizophrenia and healthy subjects. Although there is a lack of evidence to support this assumption as the methodology has not yet been applied to patients with schizophrenia, we know from our own studies in critically ill patients that there is a time-dependent increase or decrease in AChE and BChE activity in the setting of delirium or septic-associated encephalopathy. ${ }^{17} 1866$ Statistical analysis was performed using unpaired Mann-Whitney tests. Retrospective subgrouping will be performed for the secondary endpoints (group 1: initial diagnosis schizophrenia, therapy-naive until hospital admission; group 2: initial diagnosis of schizophrenia, pretherapy with medication (psychiatrically indicated drugs are relevant); group 3: known schizophrenia-another disorder that led to admission to psychiatry wards). The subgroups are further divided into patients with predominantly positive or negative symptoms. Whether patients have predominantly positive or negative symptoms is determined by the assessment of the principal investigator according to the PANSS. The statistical evaluation of the determinants will 
be conducted by multivariate analysis. Depending on the size of the subgroups, further appropriate statistical tests will be applied.

\section{Power and sample size calculation}

Table 2 shows the determinants of the sample size calculation.

The number of patients calculated for AChE activity is $\mathrm{n}=35$, while the number for BChE activity is $\mathrm{n}=21$. Thus, the higher sample size for AChE activity is authoritative. It is assumed that 10 patients $(28.5 \%)$ will drop out of the study prematurely. Therefore, we plan to include a total of $n=50$ patients with schizophrenia and $n=50$ as comparison group of healthy subjects, matched by age and sex.

\section{Ethics and dissemination}

The study was approved by the ethics committee of the University Ulm, Germany, Trial Code No. 280/20 (09.09.2020). The study and study protocol conform to the Declaration of Helsinki in its current form. Written informed consent will be obtained from all patients or another surrogate decision-maker as appropriate. For the CLASH study, we intend to publish the study plan as well as the results in a scientific journal.

\section{Author affiliations \\ ${ }^{1}$ Department of Anaesthesiology, University Hospital UIm, Ulm, Germany ${ }^{2}$ Institute of Epidemiology and Medical Biometry, University of Ulm, Ulm, Germany ${ }^{3}$ Department of Psychiatry and Psychotherapy III, University Hospital UIm, Ulm, Germany}

Correction notice This article has been corrected since it first published. Author name 'Carlos Schönfeldt-Lecuona' has been updated.

Contributors BS, EB and CS-L planned the study and wrote the manuscript in equal measure. BM carried out the sample size calculation and is supporting the study as well as the analysis of the results as a statistical expert. He also corrected the manuscript. C-LW and TH perform the measurements as doctoral students under supervision and are involved in the editing of the manuscript.

Funding The authors have not declared a specific grant for this research from any funding agency in the public, commercial or not-for-profit sectors.

Competing interests BS and EB have received lecture fees from Köhler Chemie in the past.

Patient and public involvement Patients and/or the public were not involved in the design, or conduct, or reporting, or dissemination plans of this research.

Patient consent for publication Not applicable.

Provenance and peer review Not commissioned; externally peer reviewed.

Open access This is an open access article distributed in accordance with the Creative Commons Attribution Non Commercial (CC BY-NC 4.0) license, which permits others to distribute, remix, adapt, build upon this work non-commercially, and license their derivative works on different terms, provided the original work is properly cited, appropriate credit is given, any changes made indicated, and the use is non-commercial. See: http://creativecommons.org/licenses/by-nc/4.0/.

ORCID iD

Benedikt Schick http://orcid.org/0000-0002-7988-3947

\section{REFERENCES}

1 Kahn RS, Sommer IE, Murray RM, et al. Schizophrenia. Nat Rev Dis Primers 2015;1:15067.

2 Hettige NC, Bani-Fatemi A, Sakinofsky I, et al. A biopsychosocial evaluation of the risk for suicide in schizophrenia. CNS Spectr 2018;23:253-63.
3 Crow TJ. Positive and negative schizophrenic symptoms and the role of dopamine. Br J Psychiatry 1980;137:383-6.

4 Carrà G, Crocamo $\mathrm{C}$, Angermeyer $\mathrm{M}$, et al. Positive and negative symptoms in schizophrenia: a longitudinal analysis using latent variable structural equation modelling. Schizophr Res 2019;204:58-64.

5 Soares-Weiser K, Maayan N, Bergman H. First RANK symptoms for schizophrenia. Cochrane schizophrenia group, Herausgeber. Cochrane Database of Systematic Reviews 2020.

6 Moscarelli M. A major flaw in the diagnosis of schizophrenia: what happened to the Schneider's first RANK symptoms. Psychol Med 2020;50:1409-17.

7 Brugger SP, Angelescu I, Abi-Dargham A, et al. Heterogeneity of striatal dopamine function in schizophrenia: meta-analysis of variance. Biol Psychiatry 2020;87:215-24.

8 Németh G, Laszlovszky I, Czobor P, et al. Cariprazine versus risperidone monotherapy for treatment of predominant negative symptoms in patients with schizophrenia: a randomised, doubleblind, controlled trial. Lancet 2017;389:1103-13.

9 Lau C-I, Wang H-C, Hsu J-L, et al. Does the dopamine hypothesis explain schizophrenia? Rev Neurosci 2013;24:389-400.

10 Zavitsanou K, Katerina Z, Katsifis A, et al. Investigation of m1/ $\mathrm{m} 4$ muscarinic receptors in the anterior cingulate cortex in schizophrenia, bipolar disorder, and major depression disorder. Neuropsychopharmacology 2004;29:619-25.

11 Deng C, Huang X-F. Decreased density of muscarinic receptors in the superior temporal gyrusin schizophrenia. J Neurosci Res 2005;81:883-90.

12 Dean B. Understanding the pathology of schizophrenia: recent advances from the study of the molecular architecture of postmortem CNS tissue. Postgrad Med J 2002;78:142-8.

13 Hyde TM, Crook JM. Cholinergic systems and schizophrenia: primary pathology or epiphenomena? J Chem Neuroanat 2001;22:53-63.

14 Potasiewicz A, Golebiowska J, Popik P, et al. Procognitive effects of varenicline in the animal model of schizophrenia depend on $\alpha 4 \beta 2$ - and $\alpha$ 7-nicotinic acetylcholine receptors. J Psychopharmacol 2018;33:269881118812097.

15 Nikiforuk A, Kos T, Hołuj M, et al. Positive allosteric modulators of alpha 7 nicotinic acetylcholine receptors reverse ketamineinduced schizophrenia-like deficits in rats. Neuropharmacology 2016;101:389-400.

16 Parthiban M, Rajasekaran MB, Ramakumar S, et al. Molecular modeling of human pentameric alpha(7) neuronal nicotinic acetylcholine receptor and its interaction with its agonist and competitive antagonist. J Biomol Struct Dyn 2009;26:535-47.

17 Zujalovic B, Mayer B, Hafner S, et al. AChE-activity in critically ill patients with suspected septic encephalopathy: a prospective, single-centre study. BMC Anesthesiol 2020;20:287.

18 Barth E, Bracht H, Georgieff M. AChE- und BChE-Aktivität ALS Entscheidungshilfe für die medikamentöse therapie von Delir und kognitiver Dysfunktion bei Intensivpatienten. Barth E, Bracht H, Georgieff M, Zujalovic B: AChE- und BChE-Aktivität ALS Entscheidungshilfe für die medikamentöse therapie von Delir und kognitiver Dysfunktion bei Intensivpatienten. 10. Mai 2019;52019:233-42.

19 John M, Ely EW, Halfkann D, et al. Acetylcholinesterase and butyrylcholinesterase in cardiosurgical patients with postoperative delirium. J Intensive Care 2017;5:29.

20 Yohn SE, Conn PJ. Positive allosteric modulation of $\mathrm{M}_{1}$ and $\mathrm{M}_{4}$ muscarinic receptors as potential therapeutic treatments for schizophrenia. Neuropharmacology 2018;136:438-48.

21 Gibbons A, Dean B. The cholinergic system: an emerging drug target for schizophrenia. Curr Pharm Des 2016;22:2124-33.

22 Keefe RSE, Malhotra AK, Meltzer HY, et al. Efficacy and safety of donepezil in patients with schizophrenia or schizoaffective disorder: significant placebo/practice effects in a 12-week, randomized, double-blind, placebo-controlled trial. Neuropsychopharmacology 2008;33:1217-28.

23 Lenzi A, Maltinti E, Poggi E, et al. Effects of rivastigmine on cognitive function and quality of life in patients with schizophrenia. Clin Neuropharmacol 2003;26:317-21.

24 Papke RL, Horenstein NA, Stokes C. Nicotinic Activity of Arecoline, the Psychoactive Element of "Betel Nuts", Suggests a Basis for Habitual Use and Anti-Inflammatory Activity. PLoS One 2015;10:e0140907.

25 Stryjer R, Strous R, Bar F, et al. Donepezil augmentation of clozapine monotherapy in schizophrenia patients: a double blind cross-over study. Hum Psychopharmacol 2004;19:343-6.

26 Sullivan RJ, Allen JS, Otto C, et al. Effects of chewing betel nut (Areca catechu) on the symptoms of people with schizophrenia in Palau, Micronesia. Br J Psychiatry 2000;177:174-8. 
27 Cascella M, Muzio MR, Bimonte S, et al. Postoperative delirium and postoperative cognitive dysfunction: updates in pathophysiology, potential translational approaches to clinical practice and further research perspectives. Minerva Anestesiol 2018;84:246-60.

28 Cerejeira J, Batista P, Nogueira V, et al. Low preoperative plasma cholinesterase activity as a risk marker of postoperative delirium in elderly patients. Age Ageing 2011;40:621-6.

29 Welsh TJ, van der Wardt V, Ojo G, et al. Anticholinergic drug burden Tools/Scales and adverse outcomes in different clinical settings: a systematic review of reviews. Drugs Aging 2018;35:523-38.

30 Salahudeen MS, Duffull SB, Nishtala PS. Anticholinergic burden quantified by anticholinergic risk scales and adverse outcomes in older people: a systematic review. BMC Geriatr 2015;15:31.

31 de Leon J, Dadvand M, Canuso C, et al. Schizophrenia and smoking: an epidemiological survey in a state Hospital. Am J Psychiatry 1995;152:453-5.

32 Šagud M, Vuksan-Ćusa B, Jakšić N, et al. Nicotine dependence in Croatian male inpatients with schizophrenia. BMC Psychiatry 2018;18:18.

33 Kumari V, Postma P. Nicotine use in schizophrenia: the self medication hypotheses. Neurosci Biobehav Rev 2005;29:1021-34.

34 Isuru A, Rajasuriya M. Tobacco smoking and schizophrenia: reexamining the evidence. BJPsych Adv 2019;25:363-72.

35 Leonard S, Mexal S, Freedman R. Smoking, genetics and schizophrenia: evidence for self medication. J Dual Diagn 2007;3:43-59.

36 Koukouli F, Rooy M, Tziotis D, et al. Nicotine reverses hypofrontality in animal models of addiction and schizophrenia. Nat Med 2017:23:347-54.

37 Sinkus ML, Lee MJ, Gault J, et al. A 2-base pair deletion polymorphism in the partial duplication of the alpha7 nicotinic acetylcholine gene (CHRFAM7A) on chromosome 15q14 is associated with schizophrenia. Brain Res 2009;1291:1-11.

38 Ripoll N, Bronnec M, Bourin M. Nicotinic receptors and schizophrenia. Curr Med Res Opin 2004;20:1057-74.

39 Scarr E, Dean B. Muscarinic receptors: do they have a role in the pathology and treatment of schizophrenia? J Neurochem 2008;107:1188-95.

40 Radek RJ, Kohlhaas KL, Rueter LE, et al. Treating the cognitive deficits of schizophrenia with alpha4beta2 neuronal nicotinic receptor agonists. Curr Pharm Des 2010;16:309-22.

41 Fond G, Lançon C, Korchia T, et al. The role of inflammation in the treatment of schizophrenia. Front Psychiatry 2020;11:160.

42 Buckley PF. Neuroinflammation and schizophrenia. Curr Psychiatry Rep 2019;21:72.

43 Marques TR, Ashok AH, Pillinger T, et al. Neuroinflammation in schizophrenia: meta-analysis of in vivo microglial imaging studies. Psychol Med 2019;49:2186-96.

44 Na K-S, Jung H-Y, Kim Y-K. The role of pro-inflammatory cytokines in the neuroinflammation and neurogenesis of schizophrenia. Prog Neuropsychopharmacol Biol Psychiatry 2014;48:277-86.

45 Pasternak O, Kubicki M, Shenton ME. In vivo imaging of neuroinflammation in schizophrenia. Schizophr Res 2016;173:200-12.

46 Meyer JH, Cervenka S, Kim M-J, et al. Neuroinflammation in psychiatric disorders: PET imaging and promising new targets. Lancet Psychiatry 2020;7:1064-74.

47 Pavlov VA, Tracey KJ. The vagus nerve and the inflammatory reflex--linking immunity and metabolism. Nat Rev Endocrinol 2012;8:743-54.
48 Pavlov VA, Wang H, Czura CJ, et al. The cholinergic antiinflammatory pathway: a missing link in neuroimmunomodulation. Mol Med 2003;9:125-34

49 Zivkovic AR, Schmidt K, Sigl A, et al. Reduced serum butyrylcholinesterase activity indicates severe systemic inflammation in critically ill patients. Mediators Inflamm 2015;2015:1-11.

50 Zaghloul N, Addorisio ME, Silverman HA, et al. Forebrain cholinergic dysfunction and systemic and brain inflammation in murine sepsis survivors. Front Immunol 2017;8:1673.

51 Maldonado JR. Delirium pathophysiology: an updated hypothesis of the etiology of acute brain failure. Int J Geriatr Psychiatry 2018;33:1428-57.

52 Leza JC, García-Bueno B, Bioque M, et al. Inflammation in schizophrenia: a question of balance. Neurosci Biobehav Rev 2015;55:612-26.

53 Khandaker GM, Cousins L, Deakin J, et al. Inflammation and immunity in schizophrenia: implications for pathophysiology and treatment. Lancet Psychiatry 2015;2:258-70.

54 Müller N. Inflammation in schizophrenia: pathogenetic aspects and therapeutic considerations. Schizophr Bull 2018;44:973-82.

55 Distelmaier K, Winter M-P, Rützler K, et al. Serum butyrylcholinesterase predicts survival after extracorporeal membrane oxygenation after cardiovascular surgery. Crit Care 2014;18:R24.

56 Bitzinger DI, Gruber M, Tümmler S, et al. In vivo effects of neostigmine and physostigmine on neutrophil functions and evaluation of acetylcholinesterase and butyrylcholinesterase as inflammatory markers during experimental sepsis in rats. Mediators Inflamm 2019;2019:1-12.

57 Mabrouk H, Mechria H, Mechri A, et al. [Butyrylcholinesterase activity in schizophrenic patients]. Ann Biol Clin 2011;69:647-52.

58 Zivkovic AR, Tourelle KM, Brenner T, et al. Reduced serum cholinesterase activity indicates splenic modulation of the sterile inflammation. J Surg Res 2017;220:275-83.

59 Zivkovic AR, Schmidt K, Stein T, et al. Bedside-measurement of serum cholinesterase activity predicts patient morbidity and length of the intensive care unit stay following major traumatic injury. Sci Rep 2019;9:10437.

60 Zivkovic AR, Bender J, Brenner T, et al. Reduced butyrylcholinesterase activity is an early indicator of trauma-induced acute systemic inflammatory response. J Inflamm Res 2016;9:221-30.

61 Tracey KJ. Reflex control of immunity. Nat Rev Immunol 2009;9:418-28

62 Huffman WJ, Subramaniyan S, Rodriguiz RM, et al. Modulation of neuroinflammation and memory dysfunction using percutaneous vagus nerve stimulation in mice. Brain Stimul 2019;12:19-29.

63 Schneider L, Jabrailova B, Soliman $\mathrm{H}$, et al. Pharmacological cholinergic stimulation as a therapeutic tool in experimental necrotizing pancreatitis. Pancreas 2014:43:41-6.

64 Worek F, Mast U, Kiderlen D, et al. Improved determination of acetylcholinesterase activity in human whole blood. Clin Chim Acta 1999:288:73-90.

65 038-009I_S3_Schizophrenie_2019-03.pdf [Internet]. [zitiert 11. März 2021]. Verfügbar unter. Available: https://www.awmf.org/uploads/tx szleitlinien/038-009I_S3_Schizophrenie_2019-03.pdf

66 Zujalovic B, Barth E. Delirium accompanied by cholinergic deficiency and organ failure in a 73-year-old critically ill patient: physostigmine as a therapeutic option. Case Rep Crit Care 2015;2015:1-3. 